\title{
A Novel Spectrometer for Measuring Laser-Produced Plasma $X$-Ray in Inertial Confinement Fusion
}

\author{
Zhu Gang, ${ }^{1,2}$ Xiong Xian-cai, ${ }^{2}$ Zhong Xian-xin, ${ }^{2}$ and Yang Yan ${ }^{1}$ \\ ${ }^{1}$ Chongqing University of Technology, 69 Hongguang Avenue, Chongqing 400054, China \\ ${ }^{2}$ College of Optoelectronic Engineering, Chongqing University, 174 Shazheng Avenue, Chongqing 400044, China
}

Correspondence should be addressed to Yang Yan, ahasnake@126.com

Received 12 June 2012; Accepted 18 July 2012

Academic Editor: Xing Chen

Copyright ( 2012 Zhu Gang et al. This is an open access article distributed under the Creative Commons Attribution License, which permits unrestricted use, distribution, and reproduction in any medium, provided the original work is properly cited.

\begin{abstract}
In the experimental investigations of inertial confinement fusion, the laser-produced high-temperature plasma contains very abundant information, such as the electron temperature and density, ionization. In order to diagnose laser-plasma distribution in space and evolution in time, an elliptical curved crystal spectrometer has been developed and applied to diagnose X-ray of laserproduced plasma in $0.2 \sim 2.46 \mathrm{~nm}$ region. According to the theory of Bragg diffraction, four kinds of crystal including LiF, PET, $\mathrm{MiCa}$, and KAP were chosen as dispersive elements. The distance of crystal lattice varies from 0.4 to $2.6 \mathrm{~nm}$. Bragg angle is in the range of $30^{\circ} \sim 67.5^{\circ}$, and the spectral detection angle is in $55.4^{\circ} \sim 134^{\circ}$. The curved crystal spectrometer mainly consists of elliptical curved crystal analyzer, vacuum configuration, aligning device, spectral detectors and three-dimensional microadjustment devices. The spectrographic experiment was carried out on the XG-2 laser facility. Emission spectrum of Al plasmas, Ti plasma, and $\mathrm{Au}$ plasmas have been successfully recorded by using X-ray CCD camera. It is demonstrated experimentally that the measured wavelength is accorded with the theoretical value.
\end{abstract}

\section{Introduction}

A large number of high-temperature and high-density plasmas are produced by laser irradiating the target, and the $\mathrm{X}$ ray spectra emitted from them contain very abundant information, from which the electron temperature and density can be deduced. It is also a powerful tool to investigate the plasma formation and evolution, and it plays a significant role in the inertial confinement fusion experiments [1-4]. In the recent years, many spectrographs have been developed to measure the laser-produced plasma X-ray, among them, the grating spectrographs $[5,6]$ have been used to measure $\mathrm{X}$ ray in the low energy region, and the flat and bent crystal spectrographs [7-16] have been designed to measure $\mathrm{X}$ ray in the high-energy region. Compared with the grating spectrographs, because the crystal lattice spacing of the diffracting planes is very short up to nanometer order, the crystal spectrographs can be applied to measure the shorter $\mathrm{X}$-ray by using the natural or artificial crystals as the dispersive elements. In this paper, we describe an elliptically bent crystal spectrograph with a 0.9586 eccentricity, a long $1350 \mathrm{~mm}$ focal distance, and a 30 67.5 degree Bragg angle.

\section{Optical System Design}

2.1. Elliptical Focusing Light Path. The characteristic of optical system is an elliptical geometry. The X-ray source is located at the front focal point. The X-rays diffracted by the elliptically curved crystal are focused at the rear focal point where a width-adjustable exit slit is positioned, and a piece of filter foiler is placed over the exit slit to cut off the stray light. The light path is shown in Figure 1. The X-ray CCD or streak cameras are effectively applied to simultaneously measure $\mathrm{X}$-ray spectra of a nanosecond source with the spatial and temporal resolutions on the back of the rear focal point.

The elliptical eccentricity and focal length are 0.9586 and $1350 \mathrm{~mm}$, respectively. And the whole optical path length is $1456 \mathrm{~mm}$. As the two same elliptical crystal analyzers were placed symmetrically, the upper channel is to get space resolved spectra by X-CCD, and the other channel 


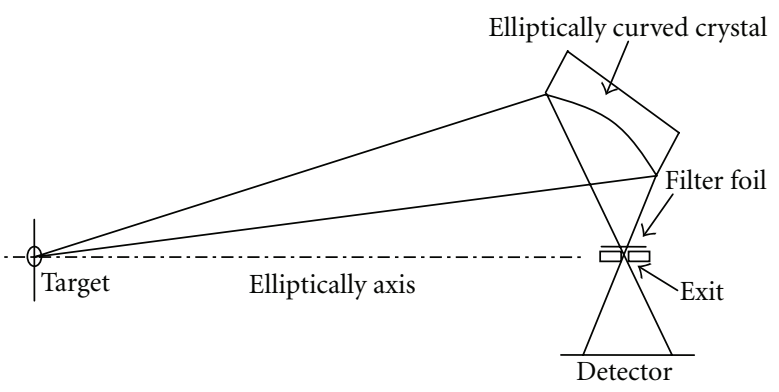

FIgURE 1: Light path of curved crystal spectrograph.

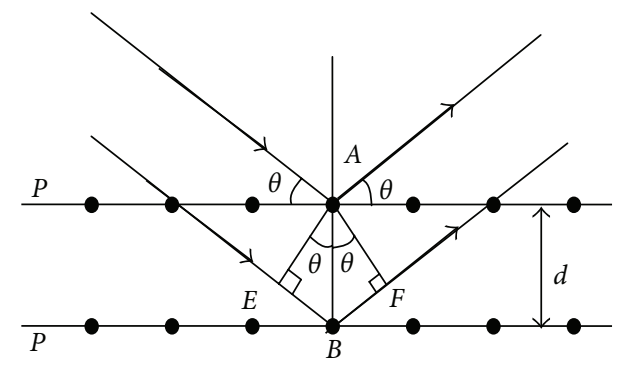

FIgURE 2: Bragg reflection.

TABLE 1: Wavelength range analyzed by four crystals.

\begin{tabular}{lccc}
\hline Crystal & $h k l$ & $2 d(\mathrm{~nm})$ & Wavelength $(\mathrm{nm})$ \\
\hline LiF & $(200)$ & 0.403 & $0.20 \sim 0.37$ \\
PET & $(002)$ & 0.874 & $0.44 \sim 0.81$ \\
Mica & $(002)$ & 1.984 & $0.99 \sim 1.83$ \\
KAP & $(1010)$ & 2.663 & $1.33 \sim 2.46$ \\
\hline
\end{tabular}

is used to measure time-resolved spectra by streak camera synchronously.

2.2. X-Ray Diffracted by the Crystal. The LiF, PET, Mica and KAP crystals have been used as dispersive elements in the elliptical spectrometer. When the X-ray is reflected by the crystal, the atoms or ions in the crystal lattice become the wave source of the subwave which emits the reflective wave in every direction, then two rows of reflective waves arouse diffraction after interference, which is shown in Figure 2.

The four crystals have different $d$ values; according to the Bragg diffraction theory, we can calculate the X-ray wavelength range measured by these crystals and illustrate in Table 1.

\section{Configuration Design}

The Curved crystal spectrometer mainly consists of elliptical curved crystal analyzer, vacuum configuration, aligning device, spectral detectors, three-dimensional microadjustment devices, and so on. The whole system is shown in Figure 3.

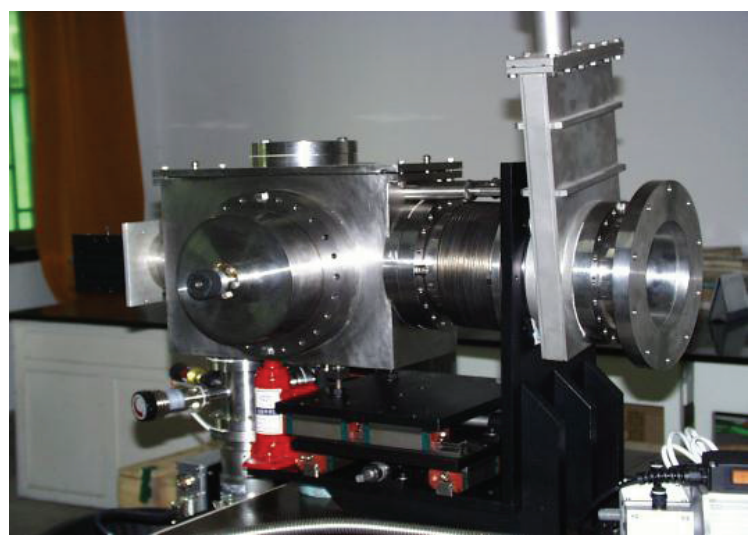

FIGURE 3: Double-channel elliptically curved crystal spectrograph.

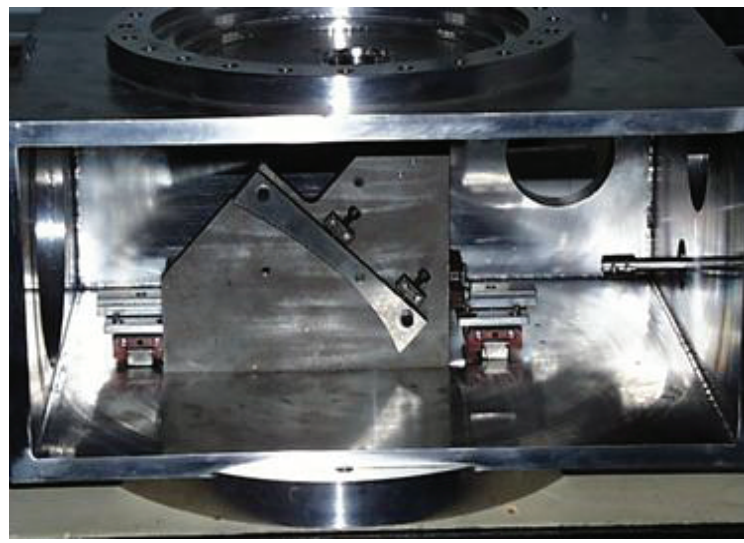

Figure 4: Elliptical curved crystal analyzer.

3.1. Elliptical Curved Crystal Analyzer. Elliptical curved crystal analyzer is the key part of spectrometer which determines the measuring region of X-ray wavelength. The analyzer of the elliptical profile was machined into a suitable substrate material using a computer-controlled milling machine. Since the peculiar technics about bending and sticking is adopted, the diffractive crystal can be fixed to the substrate material, thickness of which is just $2 \mathrm{~mm}$. The size of the elliptical substrate material is $125.64 \mathrm{~mm}$ in length and $8 \mathrm{~mm}$ in width. Elliptical curved crystal analyzer is shown in Figure 4.

3.2. Vacuum Configuration. Because the X-ray is easily absorbed in the atmosphere, the spectrometer must work in the vacuum condition. Furthermore, the X-ray steak camera can be used in the vacuum circumstance above $10^{-3} \mathrm{~Pa}$, or its photocathode imaging plane will be burned. After the spectrometer is stalled on the port of the target chamber, it is pumped by the vacuum system of the target chamber. However, the distance is about $1500 \mathrm{~mm}$ from the target to the imaging plane; due to the vacuum grade, the vacuum level does not reach to $10^{-3} \mathrm{~Pa}$ in the photocathode plane. Considering the above factors, the spectrometer has its own vacuum system, which consists of the manually operated gate valve for separating the spectrometer housing air from 
the target chamber, the vacuum meter, the molecular, and mechanical pumps through which the housing is pumped alone in spite of the atmospheric pressure in the target chamber.

3.3. Aligning Device. A small hole is drilled for aligning the analyzer to the target. The spectrometer may be rotated around the bellows in the front of the stainless-steel housing, moved in the horizontal plane through the spherical guides, and lifted through the carrier rods under the housing. In order to get a precise optical alignment for the elliptical X-ray analyzer, a laser diastimeter with $1 \mathrm{~mm}$ resolving precision is installed on the rear of the housing. Moreover, an alignment telescope is posited near the spectrometer to achieve magnifying observation of the small planar target.

A new method using the laser range finder for alignment and measurement to the spectrometer is advanced. The laser emitted from range finder thrills through the rear focal point, is reflected by surface of elliptical material, arrives the target in the end. This method can ensure the veracity and convenience during the adjustment progress.

3.4. Other Devices. Except dispersive elements, vacuum configuration, and aligning device, the curved crystal spectrometer also includes spectral detectors, and three dimensional micro-adjustment device. The spectral detector use photograph camera, CCD camera or stripe camera to obtain X-ray. The three-dimensional microadjustment device ensure that the X-ray source is located at the front focal point, and the $\mathrm{X}$-rays diffracted by the elliptically curved crystal are focused at the rear focal point where a width-adjustable exit slit is positioned.

\section{The Physical Experiment and Result Analysis}

4.1. Physical Experiment and Calculation. The spectrographic experiment was carried out on the XG-2 laser facility. The LiF, PET, Mica, and KAP crystals are adopted as the dispersive elements, which measure $\mathrm{X}$-ray in the $0.20 \sim 0.37,0.44 \sim 0.81,0.99 \sim 1.83$, and $1.33 \sim 2.46 \mathrm{~nm}$ region. The laser wavelength is $0.35 \mathrm{um}$, the laser energy is $48 \mathrm{~J}$, the pulse width is about $700 \mathrm{ps}$, and the diameter of the focal spot is about $250 \mathrm{um}$. Emission spectrum of Al plasmas, Ti plasmas, and Au plasma has been successfully recorded by using X-ray CCD camera, which are shown in Figures 5, 6, 7, and 8 .

And according to the relation between the pixel position and the X-ray wavelength, we can calculate the wavelength of the peak spectral lines. We also can get the spectral resolution by the full width at half maximum (FWHM). The calculation result is shown in Tables 2, 3, 4, and 5. They are demonstrated experimentally that the measured wavelength is accorded with the theoretical value. At the same time, experimental results show that spectral resolution of PET and KAP crystals is 956 and 1132 . Due to the vacuum below $10^{-3} \mathrm{~Pa}$ in the cathode plane of the X-ray streak camera, we did not measure the time-resolved spectrum.

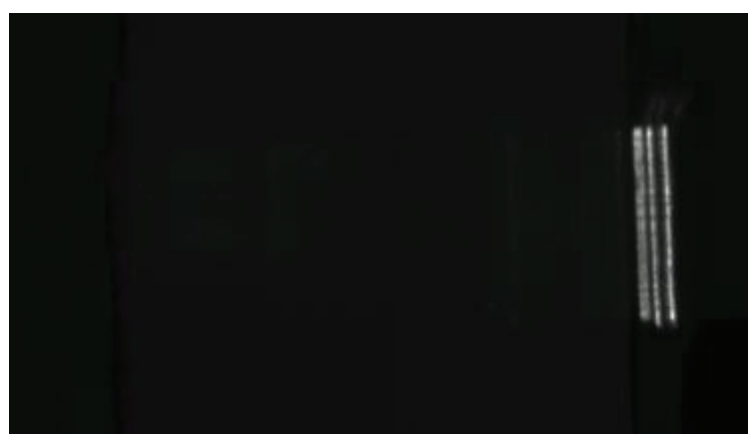

FIgURe 5: Emission spectrum of Ti plasma (LiF).

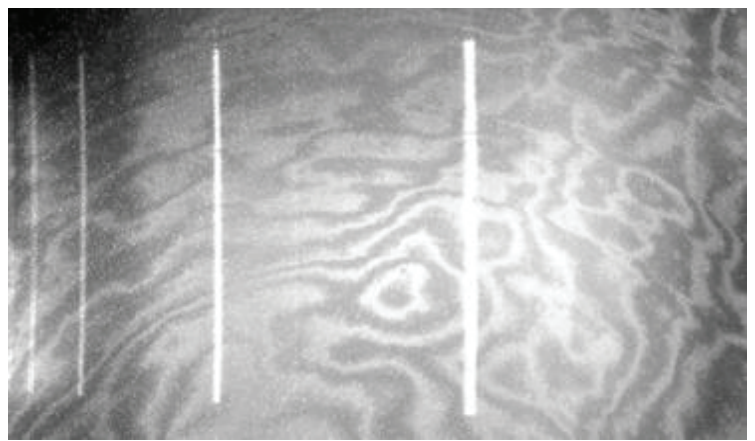

Figure 6: Emission spectrum of Al plasma (PET).

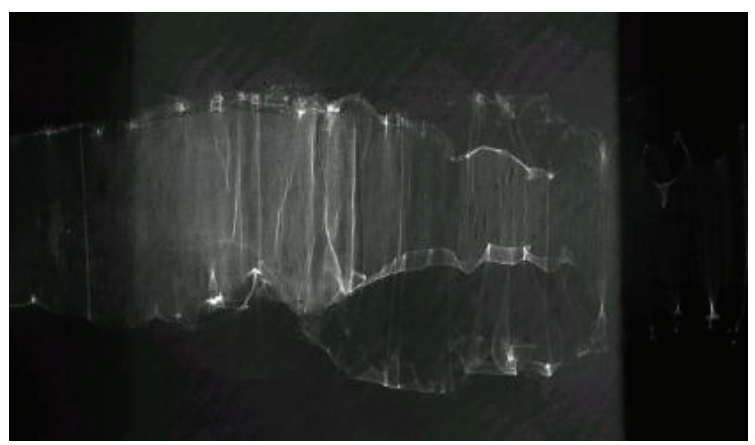

FIgURE 7: Emission spectrum of Au plasma (Mica).

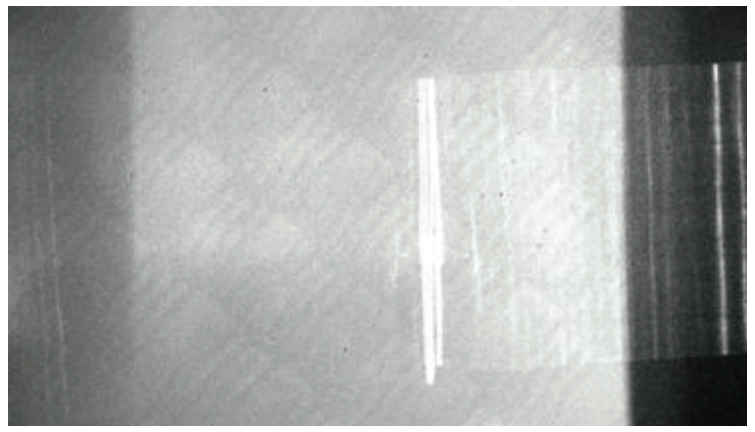

FIgURE 8: Emission spectrum of Ti plasma (KAP). 
TABLE 2: X-ray wavelength and resolution of TI plasmas.

\begin{tabular}{lcccc}
\hline Target & Crystal & Pixel & Wavelength $(\mathrm{nm})$ & Resolution \\
\hline \multirow{2}{*}{$\mathrm{Ti}$} & \multirow{2}{*}{$\mathrm{LiF}$} & 1133 & 0.2660 & 190 \\
& & 1151 & 0.2635 & 329 \\
& & 1177 & 0.2610 & 325 \\
\hline
\end{tabular}

TABLE 3: X-ray wavelength and resolution of Al plasmas.

\begin{tabular}{ccccc}
\hline Target & Crystal & Pixel & Wavelength $(\mathrm{nm})$ & Resolution \\
\hline & & 9 & 0.5605 & 701 \\
& & 56 & 0.5738 & 956 \\
$\mathrm{Al}$ & \multirow{3}{*}{ PET } & 142 & 0.5983 & 299 \\
& & 380 & 0.6629 & 368 \\
& & 834 & 0.7766 & 946 \\
\hline
\end{tabular}

TABLE 4: X-ray wavelength and resolution of Au plasmas.

\begin{tabular}{lcccc}
\hline Target & Crystal & Pixel & Wavelength $(\mathrm{nm})$ & Resolution \\
\hline \multirow{3}{*}{$\mathrm{Au}$} & \multirow{2}{*}{ Mica } & 135 & 1.0907 & 606 \\
& & 551 & 1.3495 & 750 \\
& & 627 & 1.3981 & 999 \\
\hline
\end{tabular}

TABLE 5: X-ray wavelength and resolution of Ti plasmas.

\begin{tabular}{lcccc}
\hline Target & Crystal & Pixel & Wavelength $(\mathrm{nm})$ & Resolution \\
\hline & & 761 & 2.0323 & 924 \\
& & 772 & 2.0412 & 927 \\
$\mathrm{Ti}$ & \multirow{2}{*}{ KAP } & 1150 & 2.2679 & 1132 \\
& & 1273 & 2.3187 & 682 \\
& & 1315 & 2.3356 & 730 \\
\hline
\end{tabular}

4.2. Result Analysis. From the emission spectrum of $\mathrm{Au}$ plasma which is obtained by Mica-dispersive element, we can find that the result is not so satisfied. We especially have done diffraction experiments on Mica (002) coming from Danba county using RXD-6000 diffraction instrument, and the result is shown in Figure 9. The X-ray is k $\alpha$ spectra of $\mathrm{Cu}$, and the wavelength is $0.1541874 \mathrm{~nm}$. The work voltage, current, and operation style are $40 \mathrm{kv}, 5 \mathrm{~mA}$, and $\theta-2 \theta$, respectively.

From the diffraction result, we find that the highest relatively diffraction intensity is on the 5th order not the 1st order. Then, we have observed the 3D contour of natural Mica by atom force microscope which is shown in Figure 10 and we find that there are defect and dislocation in the Mica crystal. Now, we can get a conclusion that the high-order diffraction of Mica crystal results in diffraction in several angles on elliptical curved crystal analyzer although the X-ray of Au plasma is monochromatic. So we get several spectral lines not just one line on the CCD detector. By the way, the defect and dislocation also lead the bend of spectral lines of Au plasma.

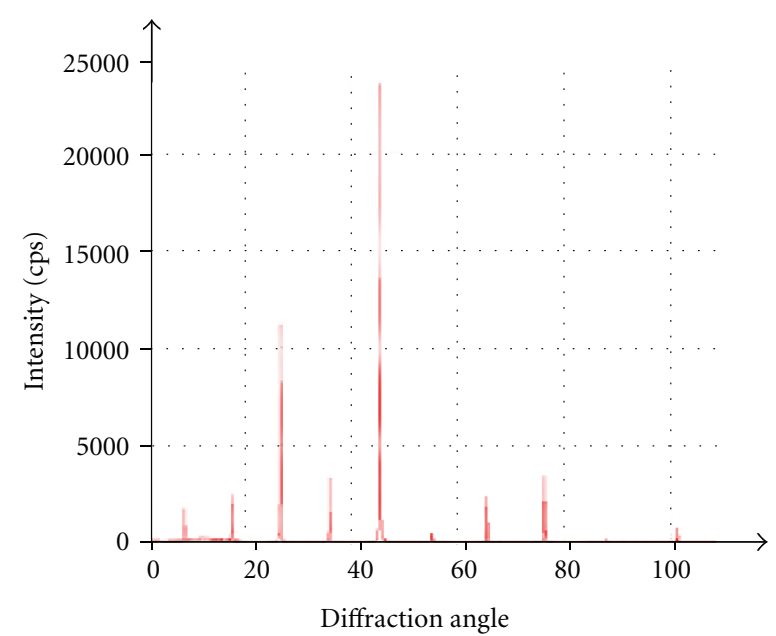

FIGURE 9: Relation between diffraction angle and relative intensity of Mica (002).

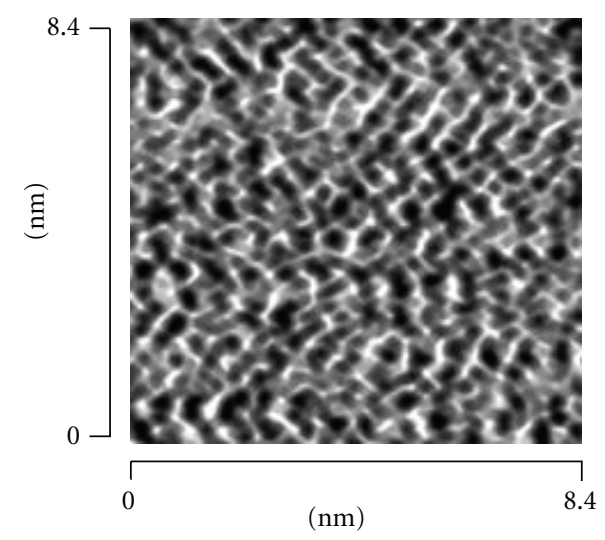

Figure 10: Contour of natural Mica.

\section{Conclusion}

We have designed and fabricated a double-channel elliptical crystal spectrometer, the important optical parameters are given in this paper, and the construction is also introduced.The spectrographic experiment was carried out on the XG-2 laser facility, and the emission spectrum of Al plasmas, Au plasma, and Ti plasmas has been successfully recorded.

\section{Acknowledgments}

This work was supported by the National Natural Science Foundation of China (51175532), by the High-Technology Research and Development Program of China (863-8043), by the Natural Science Foundation Project of CQ CSTC (key project CSTC, 2011BA4028), by the Natural Science Foundation Project of Chongqing Education Commission (CQEC, KJ120831). 


\section{References}

[1] D. K. Bradley, J. A. Delettrez, and C. P. Verdon, "Measurements of the effect of laser beam smoothing on direct drive inertial confinement fusion capsule implosions," Physical Review Letters, vol. 63, no. 6, pp. 31-38, 2003.

[2] H. B. Chen, J. Chen, B. Hilko, and E. Panarella, "Numerical comparison between the ICF and the ICF-spherical pinch," Journal of Fusion Energy, vol. 13, no. 1, pp. 45-58, 1994.

[3] M. Murakami, "Irradiation system based on dodecahedron for inertial confinement fusion," Applied Physics Letters, vol. 66, no. 13, pp. 1587-1589, 1995.

[4] F. B. Rosmej, D. H. H. Hoffmann, M. Geißel et al., "Advanced $\mathrm{x}$-ray diagnostics based on an observation of high-energy Rydberg transitions from autoionizing levels in dense laserproduced plasmas," Physical Review A, vol. 63, no. 6, Article ID 063409, 8 pages, 2001.

[5] X. Zhong, S. He, S. Chen, Y. Chen, X. Zhou, and C. He, "Grazing incidence grating spectrometer in the low energy X-ray region," in International Conference on Optoelectronic Science and Engineering, vol. 1230 of Proceedings of SPIE, pp. 567-569, August 1990.

[6] C. Fujikawa, N. Yamaguchi, T. Hara et al., "In situ calibration of a flat-field grazing incidence spectrograph with an x-ray charge-coupled device camera using a transmission grating and a laser-produced plasma for x-ray laser research," Review of Scientific Instruments, vol. 69, no. 8, pp. 2849-2853, 1998.

[7] P. Z. Fan, E. E. Fill, and G. Tietang, "A soft x-ray octadecyl hydrogen maleate crystal spectrograph," Review of Scientific Instruments, vol. 67, no. 3, pp. 786-788, 1996.

[8] B. L. Henke and P. A. Jaanimagi, "Two-channel, elliptical analyzer spectrograph for absolute, time-resolving timeintegrating spectrometry of pulsed $\mathrm{x}$-ray sources in the 100-10 000-eV region," Review of Scientific Instruments, vol. 56, no. 8, pp. 1537-1552, 1985.

[9] B. D. Shrivastava, M. S. Kushwaha, and S. K. Joshi, "Design and construction of a vacuum chamber for the $40 \mathrm{~cm}$ bent crystal x-ray spectrograph," Measurement Science and Technology, vol. 3, no. 8, pp. 723-725, 1992.

[10] Y. Aglitskiy, F. G. Serpa, E. S. Meyer et al., "The use of a spherically curved crystal spectrometer for X-ray measurements on electron beam ion trap," Physica Scripta, vol. 58, no. 2, pp. 178$181,1998$.

[11] B. S. Fraenkel and M. Bitter, "The 2D curved crystal Johansson X-ray spectrometer for diagnostics of hot plasmas," Journal of X-Ray Science and Technology, vol. 9, no. 1, pp. 29-33, 2001, Proceedings of SPIE, vol. 6794, pp. 67941W-5.

[12] T. Tchen, "Bent-crystal Bragg spectrometers with diffractional focusing for x-rays," Journal of Optics A, vol. 5, no. 1, pp. 7379, 2003.

[13] X. Xiong, X. Zhong, S. Xiao, G. Yang, and J. Gao, "Ellipticallybent crystal spectrograph for X-ray diagnosis of laser-produced plasmas," Chinese Optics Letters, vol. 2, no. 1, pp. 2728, 2004.

[14] K. Koyama, H. Tsunemi et al., "X-Ray Imaging Spectrometers(XIS) on Board Suzaku," Publications of the Astronomical Society of Japan, pp. 1-12, 2006.

[15] B. A. Mecking and G. Adams, "The CEBAF large acceptance spectrometer (CLAS)," Nuclear Instruments and Methods in Physics Research Section A, vol. 503, no. 3, pp. 513-553, 2003.

[16] J. L. Culhane, L. K. Harra, A. M. James et al., "The EUV imaging spectrometer for hinode," Solar Physics, vol. 243, no. 1, pp. 19-61, 2007. 

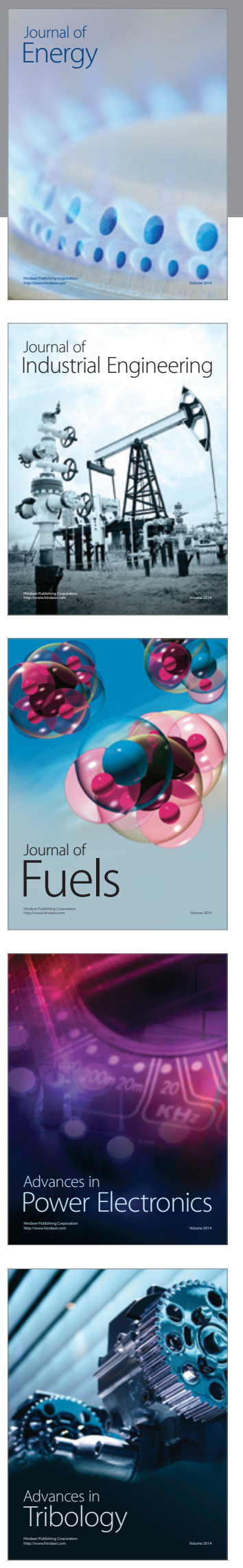
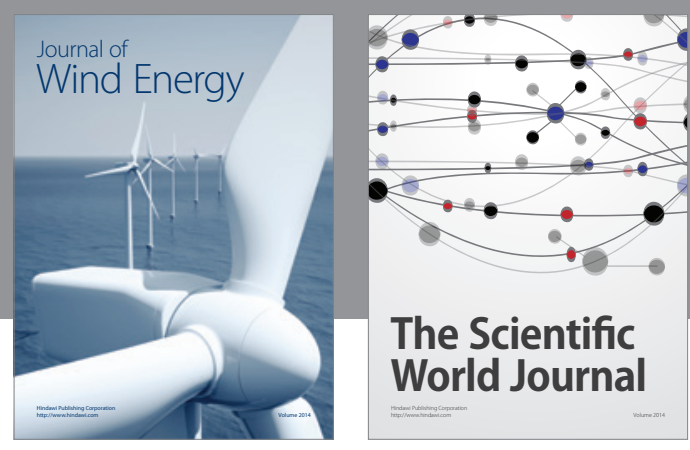

The Scientific World Journal

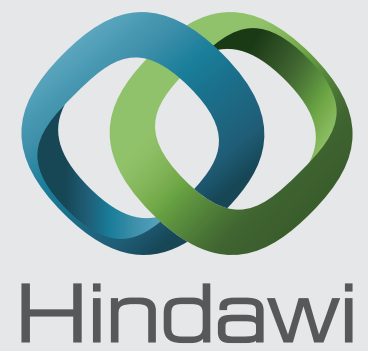

Submit your manuscripts at http://www.hindawi.com
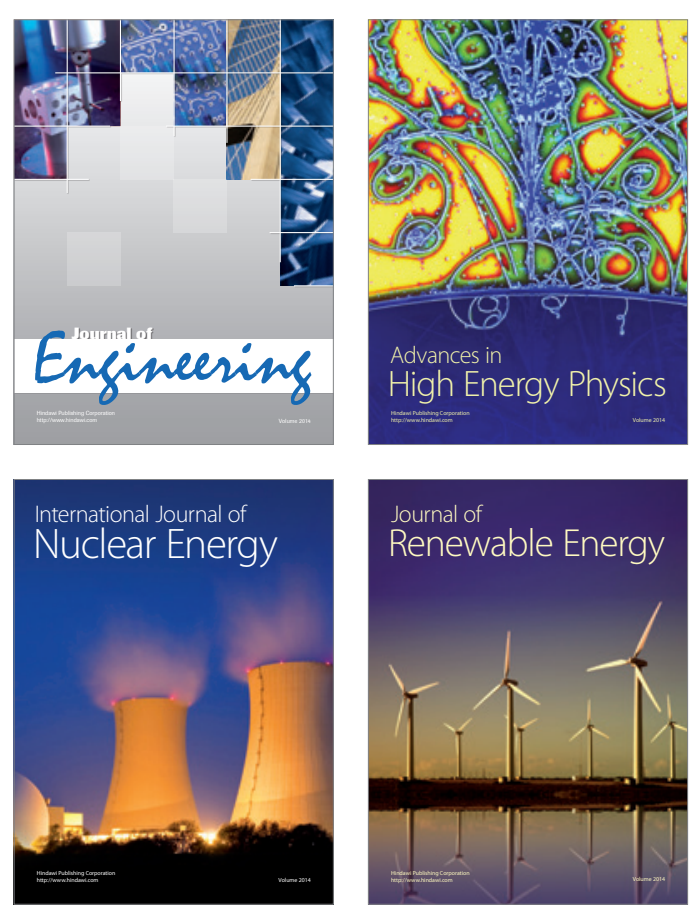

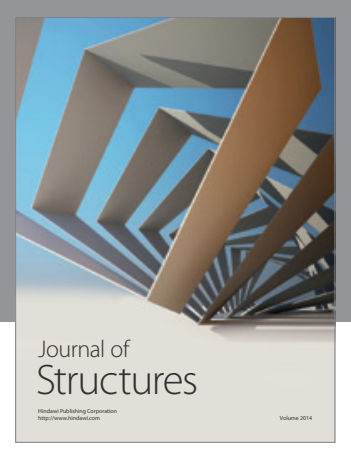

Rotating
Mechinery
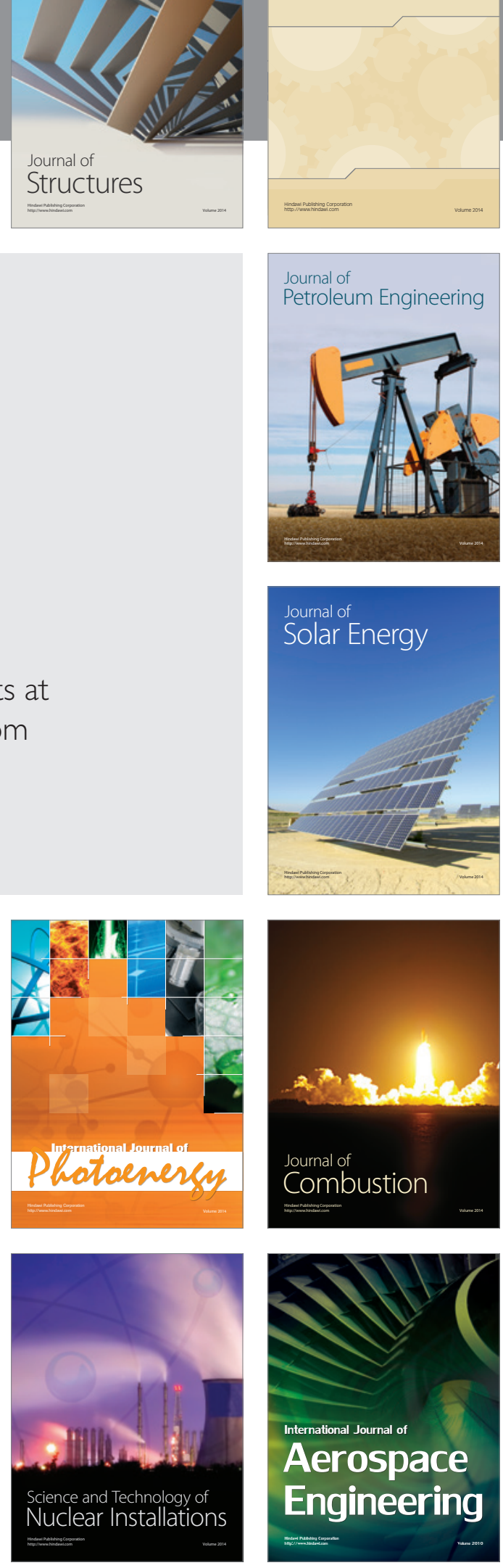